\title{
Del tabaco al uso de otras drogas: iel uso temprano de tabaco aumenta la probabilidad de usar otras drogas?
}

Ma. Elena Medina-Mora, D ra, ${ }^{(1)}$ Marco Polo Peña-C orona, $D r_{1}^{(2)}$

Patricia Cravioto, $M$ en $C,{ }^{(3)}$ Jorge Villatoro, Lic en Psic, ${ }^{(4)}$ Pablo Kuri, Dr. ${ }^{(3)}$

\begin{abstract}
Medina-Mora ME, Peña-Corona MP, Cravioto P,Villatoro J, Kuri P.

Del tabaco al uso de otras drogas: iel uso temprano de tabaco aumenta

la probabilidad de usar otras drogas?
\end{abstract}

Salud Publica Mex 2002;44 supl 1:S109-S115.

El texto completo en inglés de este artículo está disponible en: http://www.insp.mx/salud/index.html

\section{Resumen}

Objetivo.A nalizar la probabilidad de abuso de sustancias en relación con la edad de inicio del consumo de tabaco. Material y métodos. Los datos provienen de la Encuesta $\mathrm{N}$ acional de A dicciones (1998) realizada en población urbana. Resultados La edad de mayor riesgo para experimentar con sustancias es entre los 15 y los 19 años. Solamente en $5.6 \%$ de los usuarios de drogas y $13 \%$ de los que han consumido alcohol, el uso del tabaco no ocurrió primero. La probabilidad de beber en forma consuetudinaria y de presentar dependencia es mayor cuando la edad de inicio es temprana y disminuye en la medida en que se retrasa la edad de inicio. La experimentación con drogas, el continuar usándolas y el poliuso son más frecuentes entre quienes se iniciaron antes de los 15 años. Conclusiones. El inicio temprano en el consumo de tabaco incrementa la probabilidad de uso y abuso de sustancias. El texto completo en inglés de este artículo está disponible en: http://www.insp.mx/salud/ index.html

Palabras clave: inicio temprano de tabaco; uso de drogas; México
Medina-Mora ME, Peña-Corona MP,

Cravioto P,Villatoro J, Kuri P.

From tobacco to substance abuse:

does early smoking increase the

probability of substance abuse?

Salud Publica Mex 2002;44 suppl 1:S109-S115.

The English version of this paper

is available at: http://www.insp.mx/salud/index.html

\section{A bstract}

Objective. To assess the likelihood of substance abuse predicted by age of first exposure to tobacco. Material and Methods D ata from the $1998 \mathrm{~N}$ ational Household Survey on Addictions in urban areas were analyzed. Results.The age period of greater likelihood of drug experimentation is between 15 and 19 years; only $5.6 \%$ of drug users and $13 \%$ of alcohol beverage drinkers reported having experimented with drugs before trying tobacco. The probability of heavy drinking and dependence was higher at early ages of first exposure to tobacco and decreased with increasing age. Experimentation with drugs, continued use, and multiple drug use, are more frequent among smokers who started before 15 years of age. Conclusions Early to bacco use increases the likelihood of substance abuse.The English version of this paper is available at: http://www.insp.mx/salud/index.html

Key words: early tobacco exposure; drug use; Mexico

(1) Instituto $\mathrm{N}$ acional de Psiquiatría doctor Ramón de la Fuente, México, D.F., México.

(2) Coordinación General de los Institutos N acionales de Salud, Secretaría de Salud, México, D.F., México.

(3) Dirección General de Epidemiología, Secretaría de Salud, México, D.F., México.

(4) Dirección de Investigaciones Epidemiológicas y Psicosociales, Instituto Politécnico N acional, México, D.F., México.

Fecha de recibido: 26 de junio de 2001 - Fecha de aprobado: 24 de abril de 2002

Solicitud de sobretiros: Dra. Ma. Elena Medina-Mora, Calzada México Xochimilco 101, 14370 México, D.F.

Correo electrónico:medinam@ imp.edu.mx 
S e ha definido al tabaquismo como una enfermedad pediátrica debido a que la mayoría de los adultos empiezan a fumar en la adolescencia temprana. ${ }^{1}$ En México, la edad más frecuente para que los jóvenes empiecen a fumar es entre los 13 y 14 años, ${ }^{2}$ y $61 \%$ de la población adulta fumadora inicia el hábito antes de alcanzar la mayoría de edad (18 años). ${ }^{3}$ Encuestas recientes documentan también que la edad de inicio se ha disminuido pues en 1988, 57\% de los fumadores actuales o pasados habían fumado su primer cigarro antes de los 18 años, en tanto que en 1998 este índice aumentó para caracterizar a $68 \%{ }^{4}$ En total, $27.7 \%$ de la población urbana entre 12 y 65 años fuma, y $14.8 \%$ es ex fumadora y en particular $27 \%$ de los adolescentes varones de entre 12 y 17 años y $17 \%$ de las mujeres han fumado, y $15 \%$ fuman diario o casi diario.

Se sabe que la exposición temprana a drogas es un predictor significativo para el desarrollo de problemas; ${ }^{5-7}$ mientras más temprano empieza una persona a fumar, mayor será su riesgo de convertirse en un fumador regular, desarrollar dependencia y sufrir consecuencias a largo plazo derivadas de este hábito. La exposición a tabaco también se ha asociado con el riesgo de usar otras drogas, ${ }^{7}$ si bien la mayor parte de las personas que fuman tabaco nunca prueban otras drogas; así, mientras que $42 \%$ de la población urbana del país fuma, o fumó en el pasado, solamente $7.5 \%$ de los de 18 a 65 años ha usado una o más drogas, sin incluir al alcohol. ${ }^{8}$

Diferentes estudios han señalado que el tabaco constituye una droga de entrada, ${ }^{9}$ con lo cual el riesgo de usar otras drogas se incrementa. Estudios realizados en México ${ }^{10}$ documentan una importante asociación entre la conducta de consumo de tabaco y alcohol, y el consumo de drogas entre estudiantes de enseñanza media y media superior, varones y mujeres, en quienes el riesgo de probar drogas se incrementaba con la severidad del patrón de consumo, es decir, a mayor frecuencia de consumo de tabaco y cantidad de alcohol por ocasión, mayor la probabilidad de que los adolescentes prueben otras drogas.

Entre los factores que explican esta asociación están los ambientales, relacionados con las oportunidades de consumir drogas; estas son mayores para los varones que para las mujeres, sin embargo una vez que se ha tenido la oportunidad de uso se observan pocas diferencias entre uno u otro sexo. ${ }^{11}$ También se han descrito factores sociales que se relacionan con la exposición a modelos de roles durante la infancia temprana, así los hijos de padres fumadores o que beben alcohol en exceso, tienen más riesgo de fumar y usar otras drogas. ${ }^{10}$ Otros autores han sugerido que más que una exposición temprana, la exposición in utero puede predisponer a los niños a una experimentación temprana. ${ }^{12}$ Se sabe también que la probabilidad de usar drogas está asociada con factores relacionados con la disponibilidad y con el nivel de tolerancia social hacia el consumo, ${ }^{13}$ debido a que estos factores varían de una cultura a otra es importante conocer cómo se da esta asociación en México. En este artículo se analiza el riesgo de usar, abusar y desarrollar dependencia al alcohol y abusar de drogas, en relación con la edad de inicio del consumo de tabaco.

\section{Material y métodos}

Los datos provienen de la Encuesta Nacional de Adicciones de 1998 realizada en población urbana del país, en dos grupos de adolescentes entre 12 y 17 años de edad y adultos entre 18 y 65 años de edad, que habitaban hogares normales. El análisis reportado en este artículo se realizó solamente en la población adulta. El método ha sido publicado en otros reportes; ${ }^{14}$ baste mencionar que el diseño de muestra fue probabilístico estratificado, con selección de conglomerados en varias etapas de muestreo: Area Geoestadística Básica (AGEB) manzanas, viviendas e individuo. Para ello, se usó el marco cartográfico y de datos a nivel localidad y AGEB del Conteo Nacional de Población de 1995, realizado por el Instituto Nacional de Geografía, Estadística e Informática (INEGI). La información se obtuvo mediante un cuestionario estandarizado, con previa validez ${ }_{1}^{15-17}$ y aplicado cara a cara por un grupo de encuestadores entrenados.

Se utilizó un cuestionario estandarizado aplicado en estudios previos y completado en una entrevista individual. La sección sobre consumo de sustancias, incluyendo el consumo de tabaco y los problemas con los que se asocia, fue probada por diferentes países, entre ellos México, conjuntamente con la Organización Mundial de la Salud (OMS) y usadas en encuestas de

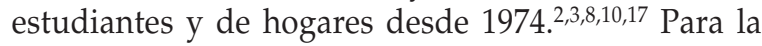
elaboración de este artículo se incluyeron las preguntas que evalúan el uso alguna vez, el número de ocasiones en que se ha consumido y la edad de inicio del uso de mariguana, cocaína, alucinógenos, heroína e inhalables; el haber fumado alguna vez, y en caso positivo, el haber fumado más de 100 cigarros y la edad de primer uso; el consumo de bebidas alcohólicas en los 12 meses previos a la encuesta, el consumo de cinco copas o más por ocasión de consumo, y los problemas relacionados con el consumo y la edad en que se consumió la primera copa completa.

Se utilizaron las definiciones de patrones de uso de la OMS; ${ }^{18}$ se definió como fumador a aquella persona que reportó haber fumado más de 100 cigarros; y uso de dro- 
gas, cuando el individuo continuaba usándolas después de haber experimentado los efectos unas cinco veces. La muestra total entrevistada fue de 5711 personas de entre 18 y 65 años de edad, con una tasa de no respuesta de $14 \%$. Con el fin de determinar si la exposición temprana a tabaco incrementaba el riesgo de usar y abusar de otras drogas se utilizó un análisis de regresión logística, en el que se incluyó la muestra total estudiada y se usó como categoría de referencia a los no fumadores.

\section{Resultados}

\section{Edad de inicio}

La edad de inicio para el consumo de tabaco, alcohol y otras drogas se presenta en la figura 1. Se analiza el porcentaje de personas que se inician en cada grupo de edad, considerando el total de consumidores de cada sustancia. Los resultados señalan que la edad más común para el inicio del consumo de cualquier droga, incluyendo el tabaco y el alcohol, es entre los 15 y los 19 años; poco más de la mitad de las personas que han usado estas sustancias inician el consumo en esta edad (60\% en el caso del tabaco, 54\% para el alcohol y $48 \%$ para cualquier otra droga). Cuando se analizan las drogas por separado se observa que la edad de inicio de la cocaína se extiende para abarcar el rango entre los 20 y los 24 años de edad (37\% se inicia entre los 15 y 19 años, y 35\% adicional entre los 20 y 24 años), mientras que la inhalación de disolventes volátiles tiene un inicio más temprano (29\% se inició antes de los 15 años, y $58 \%$ adicional entre los 15 y los 19 años).

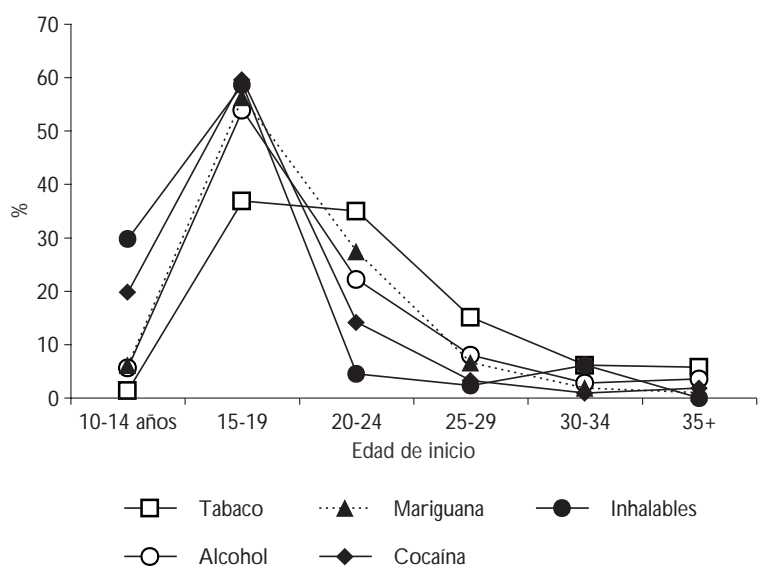

Figura 1. Edad de inicio de consumo de tabaco y de otras drogas. Encuesta Nacional de Adicciones, 1998, MÉXICO
Alrededor de las dos terceras partes de los usuarios de tabaco (80\%) y de inhalables (87\%) se iniciaron antes de los 20 años de edad; esto sólo ocurrió en $63 \%$ de los fumadores de mariguana, en $59 \%$ de los bebedores y en $38 \%$ de las personas que reportaron haber experimentado con cocaína. Solamente $5.6 \%$ de los usuarios de drogas ilegales y $13 \%$ de los que han consumido bebidas alcohólicas reportaron haber experimentado con estas sustancias antes de haber fumado tabaco.

\section{Relación entre el consumo de tabaco y el abuso y dependencia al alcohol}

En la figura 2 se presenta el índice de no fumadores y de fumadores que iniciaron el consumo en diferentes rangos de edad (antes de los 15 años, entre los 15 y 19 años, entre los 20 y 24 años y después de esta edad), que bebieron alcohol en los doce meses previos al estudio en forma consuetudinaria y que presentan diferentes problemas derivados del abuso de esta sustancia. Se definió a los fumadores como aquellas personas que reportaron haber fumado más de 100 cigarrillos en su vida; es consumo consuetudinario la ingesta de alcohol una vez por semana o con más frecuencia y el consumo se entiende como el beber cinco o más copas por ocasión con esa misma frecuencia; los indicadores de abstinencia, tolerancia y dependencia al alcohol fueron medidos de acuerdo con el Sistema de Diagnóstico en su cuarta revisión de la Asociación Psiquiátrica Norteamericana (DSM IV). ${ }^{19}$ Por ejemplo, $16 \%$ de las personas que empezaron a fumar antes de los 25 años presentaron dependencia al alcohol, en comparación con $10 \%$ de aquellos que lo hicieron después de los 25 años, y de 25 y 15\% respectivamente,

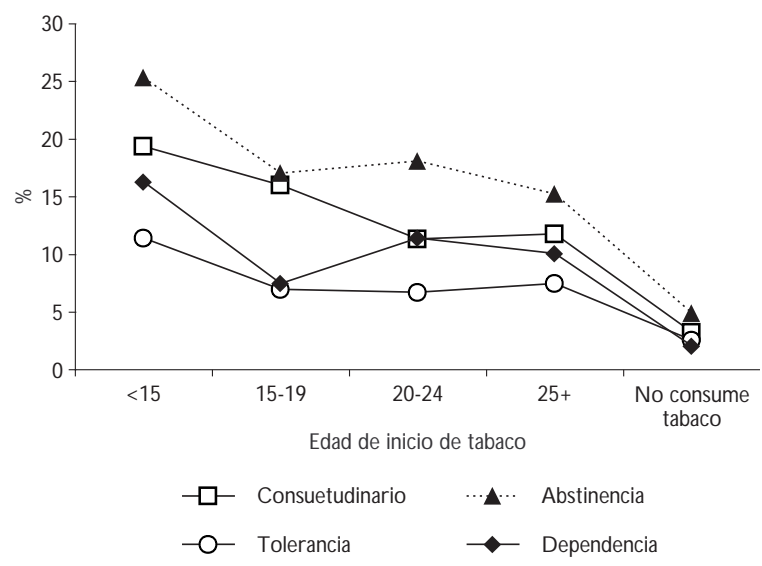

Figura 2. Relación entre edad de inicio en el CONSUMO DE TABACO Y PROBLEMAS CON EL ALCOHOL. Encuesta Nacional de Adicciones, 1998, México 
de los que manifestaron haber experimentado síntomas de abstinencia en los 12 meses previos a la encuesta. Los resultados mostraron una mayor frecuencia de problemas con el alcohol cuando el consumo de tabaco se inició antes de los 15 años de edad.

En el cuadro I se presenta esta misma información por sexo y se incluye la frecuencia y el porcentaje de personas que presentan la característica que se analiza; el complemento para la muestra total son las personas que no presentan la característica (por ejemplo, no beben o no usan drogas, estas cifras se excluyeron de los cuadros). Como puede apreciarse en el cuadro las tendencias por sexo son menos claras; entre los varones los problemas son mayores en quienes empezaron a fumar en edades tempranas, en tanto que en las mujeres los problemas se presentan en forma similar en las diferentes edades.

\section{Relación entre el consumo de tabaco y el uso de otras drogas}

La asociación entre inicio temprano en el consumo de tabaco y la experimentación con drogas legales es clara: las personas que empiezan a fumar antes de los 15 años de edad reportan mayor porcentaje de experimentación con drogas (figura 3); esta tendencia es acentuada para los varones y, en el caso de las mujeres, el bajo índice que presentan estas dos conductas dificulta llegar a conclusiones más sólidas con relación a este grupo (cuadro II).

\section{Probabilidad de usar alcohol y drogas}

La probabilidad de usar alcohol y otras drogas cuando se ha fumado se determinó mediante análisis de regresión logística. El grupo de referencia para las razones de Momios fueron los no fumadores (valor inicial de 1.00) y se compararon las probabilidades de presentar las diferentes conductas de interés en relación con la edad de inicio del consumo de tabaco. Adicionalmente, se incluyó en el análisis como variable de control a la edad del sujeto donde el grupo de referencia fue el de mayor edad, ya que las drogas en general no estaban tan disponibles para este grupo. Los resultados de este análisis se presentan en el cuadro II.

Como puede observarse para la población total la probabilidad de beber en forma consuetudinaria, y presentar dependencia al alcohol, es mayor cuando la edad de inicio es temprana, y disminuye en la medida en la que se retrasa la edad de inicio. La experimentación con drogas, el continuar usándolas después de haber experimentado con sus efectos entre una y cinco veces y el usar más de una sustancia, son significativamente más frecuentes entre aquellos fumadores que experimentaron con tabaco por primera vez antes de los 15 años.

Cuando se analizan los datos por separado para hombres y mujeres se observan en general las mismas tendencias, sin embargo, entre los varones la probabilidad de consumir alcohol en forma consuetudinaria, entre aquellos que empezaron a fumar después de los 24 años (3.16), fue mayor que para quienes lo hicieron entre los 15 y 19 años de edad (2.28), entre los 20 y 24 (1.97) años y entre los fumadores que se iniciaron antes de los 14 años (2.68).

Entre las mujeres, la probabilidad de probar drogas es mayor en aquellas que empezaron a fumar antes de los 14 años, pero la mayor probabilidad de continuar usándolas, o el beber alcohol en forma consuetudinaria lo presentan las que iniciaron el consumo entre los 15 y 19 años de edad. Contrariamente a lo esperado, la probabilidad de desarrollar dependencia se incrementa en la medida en la que aumenta la edad de inicio en el hábito tabáquico.

\section{Discusión}

Los resultados indican que la edad de mayor probabilidad para experimentar con sustancias ocurre entre los 15 y los 19 años de edad, y que solamente en $5.6 \%$ de los usuarios de drogas y $13 \%$ de los que han consumido alcohol el consumo de tabaco no ocurrió primero. También se documenta como la probabilidad de experimentar con drogas, continuar usándolas y consumir más de una, es mayor cuando la edad de inicio es temprana y disminuye en la medida en la que se retrasa la edad de inicio; estas conductas son más frecuentes entre quienes se iniciaron antes de los 15 años. De ahí que pueda concluirse que el inicio temprano en el consumo de tabaco incrementa la probabilidad de uso y abuso de sustancias.

Las mujeres muestran una conducta de consumo de otras drogas que es menos clara en relación con la edad de inicio de consumo de tabaco; en algunos casos se comportan diferente a lo que se presenta en los varones, por lo que el inicio temprano de tabaco no es siempre el factor predictor.

La relación entre inicio temprano de tabaco y el abuso de bebidas alcohólicas es menos clara, lo que indica la presencia de otros factores con mayor peso.

Los resultados de este análisis deben considerarse con cautela ya que provienen de un estudio transversal en el que se pide a los sujetos datos retrospectivos, $y$ en el que se recabaron datos mediante una muestra de conglomerados, por lo que existe el riesgo de que las varianzas estén subestimadas $y$, por tanto, los valores de $p$ pueden estar sobrestimados. Los hallazgos repor- 


\section{Cuadro I \\ EdAd de inicio del CONSUMO de tABACO y MANIFESTACIÓN DE PROBLEMA CON EL ABUSO DE ALCOHOL Y EL USO DE drogas. Encuesta Nacional de Adicciones, 1998, México}

\begin{tabular}{|c|c|c|c|c|c|c|c|c|c|c|c|c|}
\hline & & & & Edad de & 0 de & $\begin{array}{l}\text { sumo de } \\
\text { es }\end{array}$ & oaco & & & & & \\
\hline & \multicolumn{2}{|c|}{$\begin{array}{l}140 \text { menos } \\
n=304\end{array}$} & \multicolumn{2}{|c|}{$\begin{array}{c}15-19 \\
n=716\end{array}$} & \multicolumn{2}{|c|}{$\begin{array}{c}20-24 \\
n=146\end{array}$} & \multicolumn{2}{|c|}{$\begin{array}{c}25 \text { o más } \\
n=46\end{array}$} & \multicolumn{2}{|c|}{$\begin{array}{c}\text { No consume } \\
\text { tabaco } \\
n=1167\end{array}$} & \multicolumn{2}{|c|}{$\begin{array}{c}\text { Total } \\
n=2379\end{array}$} \\
\hline & $\mathrm{n}$ & $\%$ col. & $n$ & $\%$ col. & $\bar{n}$ & \% col. & $\bar{n}$ & \% col. & $\mathrm{n}$ & $\%$ col. & n & $\%$ col. \\
\hline Consumo consuetudinario & 72 & 23.7 & 148 & 20.7 & 26 & 17.6 & 11 & 23.4 & 124 & 10.7 & 381 & 16.0 \\
\hline Tolerancia & 41 & 13.3 & 62 & 8.6 & 14 & 9.5 & 4 & 7.7 & 84 & 7.2 & 204 & 8.6 \\
\hline Abstinencia & 91 & 29.9 & 145 & 20.3 & 38 & 25.9 & 8 & 17.8 & 147 & 12.6 & 429 & 18.0 \\
\hline Dependencia al alcohol & 58 & 19.1 & 67 & 9.4 & 24 & 16.2 & 5 & 10.8 & 75 & 6.4 & 228 & 9.6 \\
\hline Inhalables.Alguna vez & 23 & 7.6 & 9 & 1.2 & 2 & 1.6 & & & 11 & 0.9 & 45 & 1.9 \\
\hline Mariguana. Alguna vez & 92 & 30.3 & 115 & 16.1 & 20 & 13.6 & 2 & 4.2 & 66 & 5.7 & 295 & 12.4 \\
\hline Cocaína. Alguna vez & 29 & 9.6 & 34 & 4.8 & 3 & 2.2 & & & 19 & 1.6 & 86 & 3.6 \\
\hline \multicolumn{13}{|l|}{ Consumo de más de } \\
\hline \multicolumn{13}{|c|}{ M ujeres } \\
\hline & \multicolumn{2}{|c|}{$\begin{array}{c}14 \text { o menos } \\
n=67\end{array}$} & \multicolumn{2}{|c|}{$\begin{array}{c}15-19 \\
n=261\end{array}$} & \multicolumn{2}{|c|}{$\begin{array}{l}20-24 \\
n=89\end{array}$} & \multicolumn{2}{|c|}{$\begin{array}{c}250 \text { más } \\
n=44\end{array}$} & \multicolumn{2}{|c|}{$\begin{array}{c}\text { No consume } \\
\text { tabaco } \\
n=2881\end{array}$} & \multicolumn{2}{|c|}{$\begin{array}{c}\text { Total } \\
n=2379\end{array}$} \\
\hline & $\mathrm{n}$ & $\%$ col. & $\mathrm{n}$ & $\%$ col. & $\mathrm{n}$ & $\%$ col. & $\bar{n}$ & \% col. & $\mathrm{n}$ & $\%$ col. & $\bar{n}$ & $\%$ col. \\
\hline Consumo consuetudinario & 0 & 0.6 & 16 & 5.9 & 2 & 1.7 & & & 10 & 0.3 & 27 & 0.8 \\
\hline Tolerancia & 3 & 4.0 & 8 & 3.1 & 2 & 2.8 & 3 & 7.4 & 21 & 0.7 & 38 & 1.1 \\
\hline Abstinencia & 3 & 4.0 & 34 & 12.9 & 5 & 5.6 & 6 & 12.6 & 60 & 2.1 & 107 & 3.2 \\
\hline Dependencia al alcohol & 3 & 4.0 & 11 & 4.4 & 4 & 4.5 & 4 & 9.4 & 12 & 0.4 & 34 & 1.0 \\
\hline Inhalables. Alguna vez & & & & & & & & & 3 & 0.1 & 3 & 0.1 \\
\hline Mariguana. Alguna vez & 2 & 2.6 & 7 & 2.8 & 2 & 2.3 & 0 & 0.7 & 13 & 0.4 & 24 & 0.7 \\
\hline Cocaína. Alguna vez & 2 & 3.6 & 4 & 1.7 & 1 & 1.3 & - & - & 2 & 0.1 & 10 & 0.3 \\
\hline $\begin{array}{l}\text { Consumo de más de } \\
\text { una droga }\end{array}$ & - & - & 4 & 1.5 & 1 & 1.3 & - & - & 4 & 0.1 & 9 & 0.3 \\
\hline
\end{tabular}

Las n y los porcentajes expresan los sujetos que, de cada grupo de edad de inicio, presentan la característica analizada.

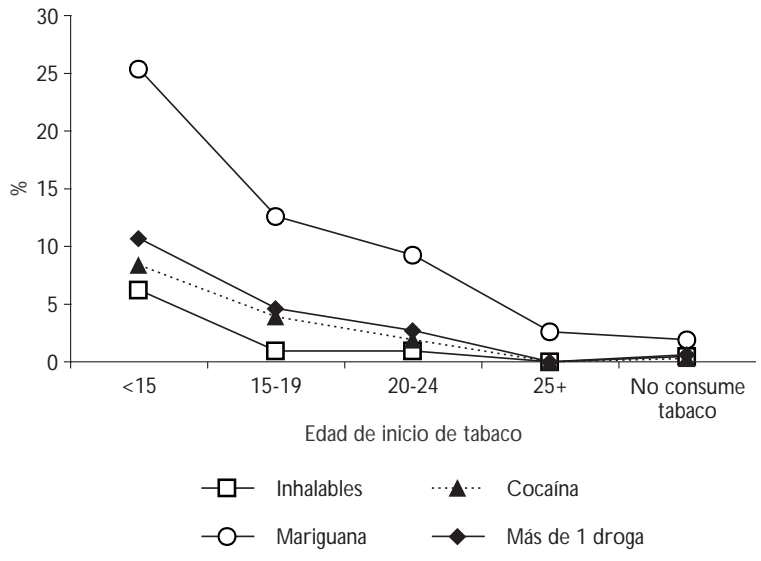

Figura 3. Relación entre edad de inicio en el CONSUMO DE TABACO Y CONSUMO DE DROGAS. ENCUESTA Nacional de Adicciones, 1998, México tados deberán confirmarse mediante estudios longitudinales, utilizando técnicas de análisis que corrijan el sesgo derivado del diseño.

Estos resultados tienen especial relevancia para la política de salud. El que las políticas de salud incluyan medidas para retrasar la edad de inicio en el consumo de tabaco en la población general, no solamente tendrá repercusiones en la disminución del índice de fumadores y de los costos para la salud, como son los derivados de la atención a la patología consecuente con esta adicción, la muerte prematura, etcétera, sino que, en algunos casos, también evitará la experimentación y el uso de otras drogas.

Es claro que este es un fenómeno complejo que requiere más estudio para identificar otros factores que intervienen en la experimentación de los jóvenes con el tabaco, como son los relacionados con la tolerancia social, la publicidad en los medios, la farmacología de la nicotina 


\section{Cuadro II

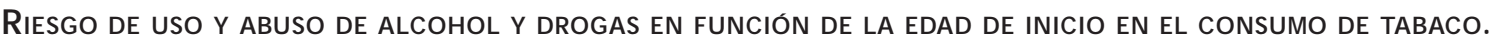 Encuesta Nacional de Adicciones, 1998, México}

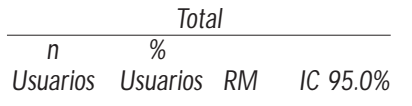

Hombres

Usuarios Usuarios RM IC $95.0 \%$ Usuarios Usuarios $\quad$ RM IC $95.0 \%$

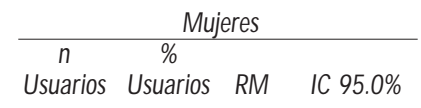

Consumo de cualquier droga

\begin{tabular}{|c|c|c|c|c|c|c|c|c|c|c|}
\hline No consume tabaco & 141 & 3.5 & 1.00 & & 95 & 8.1 & 1.00 & 47 & 1.6 & 1.00 \\
\hline \multicolumn{11}{|l|}{ Consume tabaco: } \\
\hline edad de inicio 14 o menos & 110 & 29.6 & 12.63 & $9.51-16.77$ & 101 & 33.2 & $6.184 .46-8.55$ & 9 & 13.4 & $10.304 .78-22.20$ \\
\hline Inicio 15-19 & 143 & 14.7 & 4.99 & $3.90-6.39$ & 131 & 18.3 & $2.712 .03-3.61$ & 12 & 4.6 & $3.081 .60-5.90$ \\
\hline Inicio 20-24 & 30 & 12.8 & 4.70 & $3.08-7.20$ & 24 & 16.2 & $2.501 .52-4.13$ & 7 & 7.3 & $5.802 .44-13.77$ \\
\hline Inicio 250 más & 5 & 5.2 & 2.27 & $0.87-5.92$ & 4 & 9.5 & $1.860 .66-5.22$ & 0 & 0.7 & $0.600 .02-20.19$ \\
\hline Edad actual del sujeto & 7 & & & & & & & & & \\
\hline $50-65$ & 38 & 4.4 & 1.00 & & 28 & 7.4 & 1.00 & 10 & 2.0 & 1.00 \\
\hline $18-29$ & 195 & 8.2 & 2.68 & $1.84-3.89$ & 156 & 15.5 & $2.991 .93-4.62$ & 39 & 2.9 & $1.650 .80-3.38$ \\
\hline $30-39$ & 138 & 9.1 & 2.50 & $1.70-3.66$ & 120 & 20.5 & $3.622 .32-5.66$ & 17 & 1.9 & $0.890 .40-1.98$ \\
\hline $40-49$ & 59 & 6.0 & 1.55 & $1.01-2.39$ & 51 & 12.2 & $1.921 .17-3.16$ & 8 & 1.4 & $0.68 \quad 0.26-1.77$ \\
\hline
\end{tabular}

Uso continuado de drogas

\begin{tabular}{|c|c|c|c|c|c|c|c|c|c|}
\hline No consume tabaco & 42 & 1.0 & 1.00 & 32 & 2.7 & 1.00 & 10 & 0.3 & 1.00 \\
\hline \multicolumn{10}{|l|}{ Consume tabaco: } \\
\hline edad de inicio 140 menos & 49 & 13.2 & $15.8410 .28-24.41$ & 49 & 16.1 & $7.414 .62-11.87$ & - & - & $0.010 .00-N D$ \\
\hline Inicio $15-19$ & 58 & 5.9 & $6.35 \quad 4.23-9.54$ & 53 & 7.5 & $3.051 .94-4.80$ & 5 & 1.7 & $5.511 .77-17.14$ \\
\hline Inicio 20-24 & 12 & 5.0 & $5.80 \quad 2.97-11.32$ & 10 & 6.7 & 2.91 1.37-6.17 & 2 & 2.4 & $7.921 .70-36.88$ \\
\hline Inicio 250 más & 1 & 0.4 & $0.52 \quad 0.02-16.53$ & - & - & $0.020 .00-\mathrm{ND}$ & 0 & 0.7 & $3.020 .08-110.80$ \\
\hline
\end{tabular}

Edad actual del sujeto

\begin{tabular}{|c|c|c|c|c|c|c|c|c|c|c|}
\hline $50-65$ & 11 & 1.3 & 1.00 & & 11 & 2.8 & 1.00 & 1 & 0.1 & 1.00 \\
\hline $18-29$ & 74 & 3.1 & 3.35 & $1.74-6.44$ & 67 & 6.6 & $3.081 .57-6.02$ & 7 & 0.5 & $10.370 .27-393.48$ \\
\hline $30-39$ & 50 & 3.3 & 2.93 & $1.50-5.73$ & 45 & 7.8 & 3.08 1.54-6.15 & 4 & 0.5 & $7.390 .19-292.07$ \\
\hline $40-49$ & 26 & 2.6 & 2.38 & $1.15-4.92$ & 21 & 5.1 & $2.080 .97-4.45$ & 4 & 0.8 & $12.620 .32-497.50$ \\
\hline
\end{tabular}

Poliuso

\begin{tabular}{|c|c|c|c|c|c|c|c|c|c|}
\hline No consume tabaco & 29 & 0.7 & 1.00 & 25 & 2.1 & 1.00 & 4 & 0.1 & 1.00 \\
\hline 140 menos & 40 & 10.7 & $18.7311 .39-30.80$ & 40 & 13.1 & $7.854 .64-13.29$ & - & - & $0.000 .00-N D$ \\
\hline Inicio $15-19$ & 44 & 4.5 & $7.15 \quad 4.43-11.53$ & 40 & 5.6 & $3.061 .83-5.13$ & 4 & 1.5 & $12.122 .96-49.61$ \\
\hline Inicio 20-24 & 6 & 2.7 & $5.242 .18-12.57$ & 5 & 3.6 & $2.300 .87-6.08$ & 1 & 1.3 & $16.551 .99-137.43$ \\
\hline Inicio 25 o más & - & - & $0.08 \quad 0.00-N D$ & - & - & $0.020 .00-\mathrm{ND}$ & - & - & $0.010 .00-N D$ \\
\hline
\end{tabular}

Edad actual del sujeto

\begin{tabular}{|c|c|c|c|c|c|c|c|c|c|c|c|}
\hline $50-65$ & 8 & 1.0 & 1.00 & & 8 & 2.2 & 1.00 & - & - & 1.00 & \\
\hline $18-29$ & 67 & 2.8 & 3.98 & $1.91-8.29$ & 60 & 6.0 & $3.531 .67-7.43$ & 7 & 0.5 & ND & $0.00-N D$ \\
\hline $30-39$ & 34 & 2.3 & 2.58 & $1.19-5.56$ & 32 & 5.4 & $2.661 .22-5.81$ & 2 & 0.3 & 1087.48 & $0.00-\mathrm{ND}$ \\
\hline $40-49$ & 9 & 0.9 & 1.06 & $0.41-2.75$ & 9 & 2.2 & $1.080 .41-2.81$ & - & - & 0.98 & $0.00-\mathrm{ND}$ \\
\hline
\end{tabular}

Consumo diario de alcohol

Edad de inicio del tabaco

\begin{tabular}{|c|c|c|c|c|c|c|c|c|c|}
\hline No consume tabaco & 134 & 3.3 & 1.00 & 124 & 10.7 & 1.00 & 10 & 0.3 & 1.00 \\
\hline \multicolumn{10}{|l|}{ Consume tabaco: } \\
\hline edad de inicio 140 menos & 73 & 19.6 & $7.33 \quad 5.37-10.00$ & 72 & 23.7 & $2.681 .94-3.72$ & 0 & 0.6 & $1.910 .09-42.66$ \\
\hline $15-19$ & 164 & 16.8 & $6.07 \quad 4.77-7.74$ & 148 & 20.7 & $2.281 .75-2.96$ & 16 & 5.9 & $18.518 .19-41.86$ \\
\hline
\end{tabular}




\begin{tabular}{llllllllllll}
\hline $20-24$ & 27 & 11.5 & 4.23 & $2.72-6.58$ & 26 & 17.6 & 1.97 & $1.22-3.16$ & 2 & 1.7 & $5.721 .00-32.75$ \\
\hline 25 o más & 11 & 11.9 & 4.91 & $2.50-9.64$ & 11 & 23.4 & 3.16 & $1.53-6.54$ & - & - & $0.04 \quad \mathrm{ND}-\mathrm{Nd}$ \\
\hline
\end{tabular}

\begin{tabular}{|c|c|c|c|c|c|c|c|c|c|c|}
\hline Edad actual del sujeto & & & 0.00 & $0.00-0.00$ & & & & & & \\
\hline $50-65$ & 58 & 6.8 & 1.00 & & 54 & 14.4 & 1.00 & 4 & 0.8 & 1.00 \\
\hline $18-29$ & 176 & 7.4 & 1.47 & $1.06-2.03$ & 164 & 16.3 & $1.411 .00-1.99$ & 12 & 0.9 & $1.180 .37-3.76$ \\
\hline $30-39$ & 120 & 7.9 & 1.31 & $0.94-1.84$ & 110 & 18.8 & $1.481 .03-2.13$ & 10 & 1.1 & $1.090 .33-3.56$ \\
\hline $40-49$ & 54 & 5.5 & 0.85 & $0.58-1.26$ & 53 & 12.7 & $0.890 .59-1.35$ & 1 & 0.2 & $0.26 \quad 0.03-2.04$ \\
\hline
\end{tabular}

Con dependencia al alcohol

Edad de inicio del tabaco

\begin{tabular}{|c|c|c|c|c|c|c|c|c|c|c|}
\hline No consume tabaco & 87 & 2.1 & 1.00 & & 75 & 6.4 & 1.00 & 12 & 0.4 & 1.00 \\
\hline \multicolumn{11}{|l|}{ Consume tabaco: } \\
\hline edad de inicio 140 menos & 61 & 16.4 & 0.11 & $0.08-0.15$ & 58 & 19.1 & $0.280 .19-0.41$ & 3 & 4.0 & $\begin{array}{ll}0.10 & 0.03-0.38\end{array}$ \\
\hline $15-19$ & 78 & 8.0 & 0.24 & $0.18-0.34$ & 67 & 9.4 & $0.64 \quad 0.45-0.91$ & 11 & 4.4 & $0.090 .04-0.21$ \\
\hline $20-24$ & 28 & 11.7 & 0.15 & $0.09-0.24$ & 24 & 16.2 & $0.330 .20-0.55$ & 4 & 4.5 & $0.08 \quad 0.02-0.25$ \\
\hline 250 más & 9 & 10.1 & 0.16 & $0.08-0.33$ & 5 & 10.8 & $0.480 .18-1.27$ & 4 & 9.4 & $0.03 \quad 0.01-0.11$ \\
\hline
\end{tabular}

Edad actual del sujeto

\begin{tabular}{|c|c|c|c|c|c|c|c|c|c|c|}
\hline $50-65$ & 39 & 4.6 & 1.00 & & 32 & 8.5 & 1.00 & 7 & 1.5 & 1.00 \\
\hline $18-29$ & 110 & 4.6 & 0.70 & $0.48-1.04$ & 96 & 9.6 & $0.720 .47-1.11$ & 13 & 1.0 & $0.830 .30-2.30$ \\
\hline $30-39$ & 77 & 5.1 & 0.77 & $0.51-1.16$ & 64 & 11.0 & $0.710 .45-1.12$ & 13 & 1.4 & $0.820 .31-2.19$ \\
\hline $40-49$ & 37 & 3.8 & 1.13 & $0.71-1.81$ & 36 & 8.5 & $0.960 .58-1.59$ & 1 & 0.2 & $6.290 .89-44.60$ \\
\hline
\end{tabular}

Las n y los porcentajes expresan los sujetos que, de cada grupo de edad de inicio, presentan la característica analizada

y sus efectos en el cerebro de los adolescentes y las modificaciones a nivel celular, entre otros.

\section{Referencias}

1. Giovino GA. Epidemiology of tobacco use among US adolescents. N icotine Tobacco Res 1999; 1:31-40.

2. Berenzon S, Medina-Mora ME,Villatoro J. Encuestas en estudiantes. En:, ed. El consumo de tabaco en México y encuesta nacional de adicciones 1998 (Tabaco). México, D.F. Secretaría de Salud, 2000: 61-64.

3.Tapia-C onyer R, Cravioto P, Medina-Mora ME, Hoy MJ, Kuri P. Consumo y abandono del uso de productos de tabaco. En:, ed. El consumo de tabaco en México y Encuesta N acional deAdicciones 1998 (Tabaco). México, D.F.: Secretaría de Salud, 2000: 53-59.

4. Medina-Mora ME, N atera G, Borges G, C ravioto P, Fleiz C, Tapia-C onyer R. D el siglo XX al tercer milenio. Las adicciones y la salud pública: drogas, alcohol y sociedad. Salud Mental 2001; 24(4): 3-19.

5. Anthony JC, Petronis KR. Early onset drug use and risk of later drug problems. DrugAlcohol Depend 1995; 40:9-15.

6. Grant BF. Age at smoking onset and its association with alcohol consumption and DSM-IV alcohol abuse and dependence: Results from the national longitudinal alcohol epidemiologic survey. J Subst Abuse 1998;10:163-173.

7. Hanna E, G rant BF. Parallels to early onset alcohol use in the relationship of early onset smoking with drug use and DSM-IV drug and depressive disorders: Findings from the national longitudinal epidemiologic survey. Alcohol Clin Exp Res 1999;23(3):513-522.
8. Secretaría de Salud 1998. México, D.F.: Secretaría de Salud, 1999. 9. Kandel $D$,Yamaguchi $K$, Chen K. Stages of progression in drug involvement from adolescence to adulthood: Further evidence for the gatew ay theory. J Stud Alcohol 1992; 53:447-457.

10.Villatoro J, Medina-Mora ME, Berenzon S, Juárez F, Rojas E, C arreño S. D rug use pathways among high school students of Mexico.Addiction 1998; 93(10): 1577-1588

11. Van Ethen ML, N eumark YD, Anthony JC. Male-female differences in the earliest stages of drug involvement. Addiction 1999;94(9):1413-1419. 12. Cornelius MD, Leech SL, Goldschmidt L, Day N L. Prenatal tobacco exposure: Is it a risk factor for early tobacco experimentation? N icotine Tobacco Res 2000;2:45-52.

13. Medina-Mora ME. Los factores que se relacionan con el inicio, el uso continuado y el abuso de sustancias psicoactivas en adolescentes mexicanos. Gac Med Mex 1995; 131:383-387.

14. Berumen y asociados. Reporte metodológico.Tercera Encuesta $\mathrm{N}$ acional de Adicciones. México D.F.: casa ed, 1998.

15. Medina-Mora ME, Gómez-Mont F, Campillo-Serrano C. Validity and reliability of a high school drug use questionnaire among Mexican students. Bull N arc 1981; 33:67-76.

16.Tapia-C onyer R, Medina-Mora ME, Sepúlveda J, D e la Fuente R, Kumate J. La Encuesta $\mathrm{N}$ acional de Adicciones en México. Salud Publica Mex 1990;32(5):507-522.

17.W orld Health 0 rganization. Guidelines for controling and monitoring the tobacco epidemic. Tobacco and health programme. Ginebra: W HO, 1998.

18. A merican Psychiatric Association. Diagnostic and statistical manual of mental disorders. 4th ed. W ashington, D.C.: American Psychiatric Association, 1994. 